Bioscientia Medicina: Journal of Biomedicine \& Translational Research

Journal Homepage: www.bioscmed.com

\title{
A Rare Case of Juvenile Xanthogranuloma in 8 Months Old Baby with Dyslipidemia
}

\section{Jessica Herlianez Saiful1* ${ }^{*}$ Rina Gustia²}

1,2Dermatology and Venereology Department, Faculty of Medicine, Andalas University/ Dr. M Djamil Hospital, Padang, Indonesia

\section{A R T I C L E I N F O}

\section{Keywords:}

Juvenile Xanthogranuloma

Dyslipidemia

Histiocytosis

\section{*Corresponding author:}

Jessica Herlianez Saiful

\section{E-mail address:}

\section{herlianez@gmail.com}

All authors have reviewed and approved the final version of the manuscript.

https://doi.org/10.32539/bsm.v5i4.412

\begin{abstract}
A B S T R A C T
Background: Juvenile xanthogranuloma (JXG) is a non-Langerhans cell histiocytosis disease of childhood. But due to rarity of non Langerhans cell hystiosis itself, the exact prevalence of juvenile xanthogranuloma remain unknown with only a few epidemiological journal ever published. Juvenile xanthogranuloma usually wihout lipid abnormality and systemic involvement. But association between JXG and lipid abnormalities is still not well understood. We describe a patient with multiple cutaneous JXG who also developed hyperlipidemia. Case: A case of a 8 months-old baby patient with juvenile xanthogranuloma is reported. Patient parents noticed yellowish dots on child's face since six months ago, and it was gradually increase in size and number, and spread to trunks, upper and lower limb since 2 months ago. Patients got formula milk since 7 months ago. Patients father has uncontrolled hypercholesterolemia, and grandparents had controlled dyslipidemia. Dermatological state showed yellowish plaque and papule on the face, trunk, lower limb, and upper limb. Dermoscopy show yellowish papule with sun setting appearance and branched and linear vessel on orange yellow background. Laboratory finding showed elevated lipid serum. Foam cell and Touton giant cell is found on histopathology examination. Discussion: The presented case demonstrates that skin lesions in patients with diagnosed JXG may have a variable clinical presentation, ranging from single to diffuse skin lesions, also present from the birth to childhood. The diagnosis requires histopathological confirmation to avoid misdiagnosis of malignant disease. Association between JXG and lipid abnormalities remain unknown, with most of the patient show normal lipid serum. Majority of patients presenting lesions limited to the skin requires only a strict dermatological observation.
\end{abstract}

\section{Introduction}

Juvenile xanthogranuloma (JXG) is a non langerhans cell hystiosis skin disease, most often affecting infants and young children. There is a male predominance of approximately $1.5: 1$. Cutaneous lesions usually resolve and most patients have an otherwise unremarkable course. 1,2The term "juvenile xanthogranuloma" was suggested by Helwig and Hackney in 1954 based upon histologic findings of Xanthomatous Disorders of Langerhans Cells and Macrophages histiocytes and giant cells. However, multiple cases of JXG, under different names, had previously been reported during the first half of the twentieth century. The first case of JXG was actually reported in 1905 by Adamson, who named the disorder "congenital xanthoma multiplex". ${ }^{3}$

Juvenile xanthogranuloma is a histiocytic disease of childhood. The exact prevalence is unkown, only a few epidemiological journal about juvenile xanthogranuloma ever published. The cause of JXG is not known; however, it is often suggested that the condition is reactive with histiocytes possibly responding to a traumatic or infectious stimulus. The reason for the progressive lipidation of histiocytes in the absence of hyperlipidemia is not clear. ${ }^{5}$ However, it has been shown that in adult patients with xanthogranuloma, the uptake of low-density lipoprotein and the synthesis of cholesterol within macrophages is increased34. As discussed in the section on benign cephalic histiocytosis, several authors have suggested that generalized eruptive histiocytoma, benign cephalic histiocytosis, and JXG may represent different expressions of the same 
disorder. 6

Gianotti and Caputo described two common clinical variants of JXG: a small nodular form and a large nodular form. Patients with the small nodular form, also known as the micronodular form, can present with multiple pink to red-brown, dome-shaped papules, 2$5 \mathrm{~mm}$ in diameter. ${ }^{7}$

The lesions are widely scattered on the upper part of the body and rapidly become yellow. In contrast, the more common large nodular form is characterized by one or a few nodules $1-2 \mathrm{~cm}$ in diameter. The two forms may coexist. The most common location for JXG is the head and neck followed by the upper torso, the upper extremities, and then the lower extremities. Oral JXG is rare and usually presents as a solitary yellow nodule on the lateral aspect of the tongue or the midline of the hard palate. Unusual morphologic presentations include keratotic, pedunculated,subcutaneous, clustered, plaque-like, and giant lesions. ${ }^{8}$

\section{Case Report}

An 8 months old infant was referred from pediatric department to outpatient clinic dermatology M Djamil Padang on September 11th 2019 with suspect Xanthoma with Chief complaint yellowish papule on the face that was increased in size and number and spread since 6 months ago, Initially, six months ago, Patient parent notice that patient had yellowish dots on the face that felt no itchy nor pain. This yellowish dots on the face started to increased in number and size. At this point, parents didn't get any medication for patient.

Five months ago, the yellowish dots spread to the trunk. Parents also notice that yellowish dots spread to both ears, upper limb and lower limb. Parents then took patient to dermatologist, and was diagnosed with milia, and encouraged to get laser treatment, but parent refused.

Parents took patient to another dermatologist in local hospital and was diagnosed with xanthoma. But parents was adviced to refer the patient to private hospital in the region, since there is no available medication in local hospital.

A month ago, patient was taken to dermatologist in private hospital in the region, but directly got referred to pediatric department. Patient was diagnosed with xanthoma, and was given pulveres and ointment that was applied twice a day, but the symptomps persisted. Patient then referred to pediatric department M Djamil Hospital, since there is no metabolic endocrine pediatric specialist in patients town.

Patient was diagnosed suspect xanthoma by pediatric department $\mathrm{M}$ djamil hospital and was planned to get lipid serum examination and then referred to dermatology venereology department.There was no history of applying topical traditional medication. History of decrease appetite in the last six month was denied. Patient drink formula milk since age one month. The patient had no history of yellowish dots on any part of the body before 8 months ago. Family illness history. There was no family history with yellowish papule in bodies. Patients father have high total cholesterol. Patient grandparents is known to have hypertension and dyslipidemia and controlled regularly to internal medicine department. Patient is the youngest from two sibling.

On physical examination, vital sign and another physical examination was in normal limit Dermatological state showed yellowish plaque and papule on the face, trunk, lower limb, and upper limb. Dermoscopy show yellowish papule with sun setting appearance and branched and linear vessel on orange yellow background. Patients was sent for complete blood workup and biopsied for histhopathological examination. Laboratory findings revealed elevated total cholesterol at $188 \mathrm{mg} / \mathrm{dL}$ and LDL-C at 137 $\mathrm{mg} / \mathrm{dL}$, and trigliserida at $136 \mathrm{mg} / \mathrm{dl}$ with normal liver function test results This patient was consulted to pediatrician and was diagnosed with hyperlipidemia and treated with cholestiramin sach twice a day. histopathology examination showed Accumulation of histiocytes cell in dermis, cell consists of histiocyte cell with spindle type nuclear, a group of foam cell with Touton Giant cell, with infiltrate of eosinophil and lymphocyte. This findings can be found in Juvenile Xanthogranuloma. Based on anamnesis, physical findings, dermoscopy and Histhopathology findings, we diagnosed this patient with Juvenile xanthogranuloma. 
For general treatment on this patient, we educated patients family about the disease, etiology, course illness, treatment and complication of the disease. We encourage parents to check blood cholesterol for familial hypercholesterolemia screening. We also encourage the parents to always keep an eye of the baby skin and avoid the baby from scratching the dots and bumps. Explain to the patient that the lesion will regress spontaneously and the patient has to be examined regularly in dermatology clinic. We planned for strict observation on this patient for two years. Unfortunately, the patient did not come for follow up.
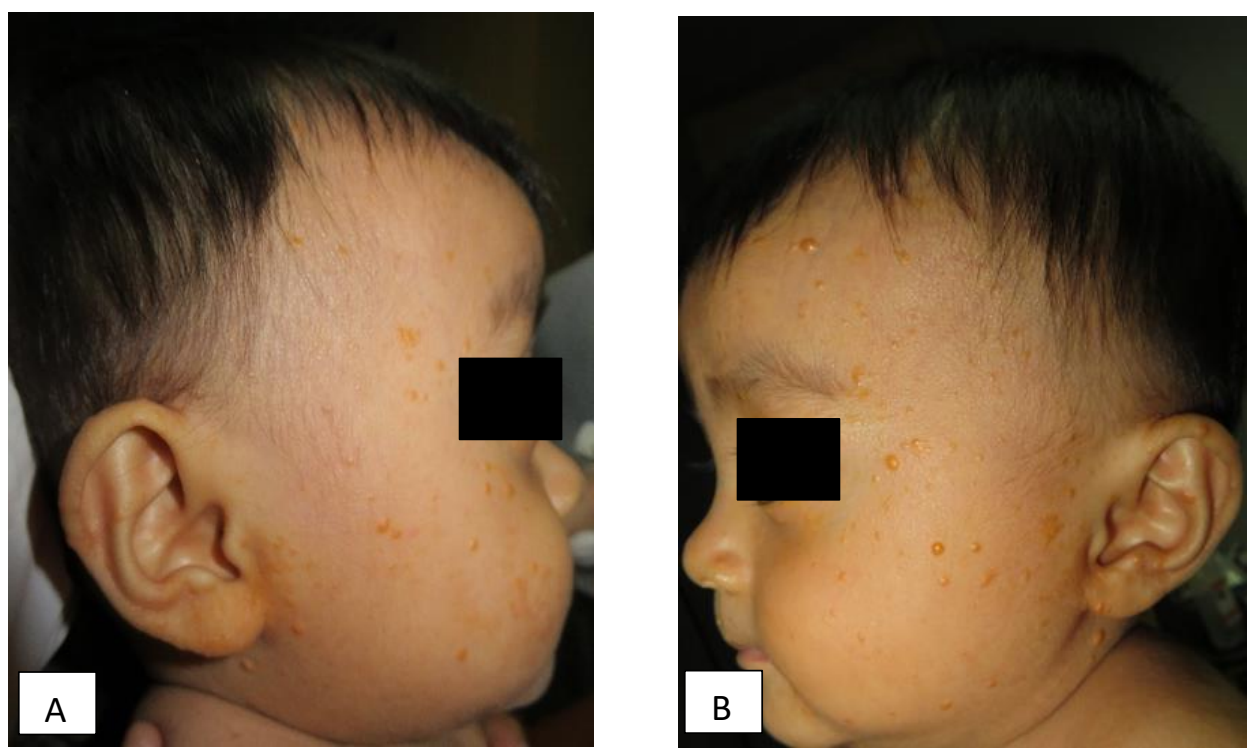

Figure 1. A, B Yellowish papule on patient face
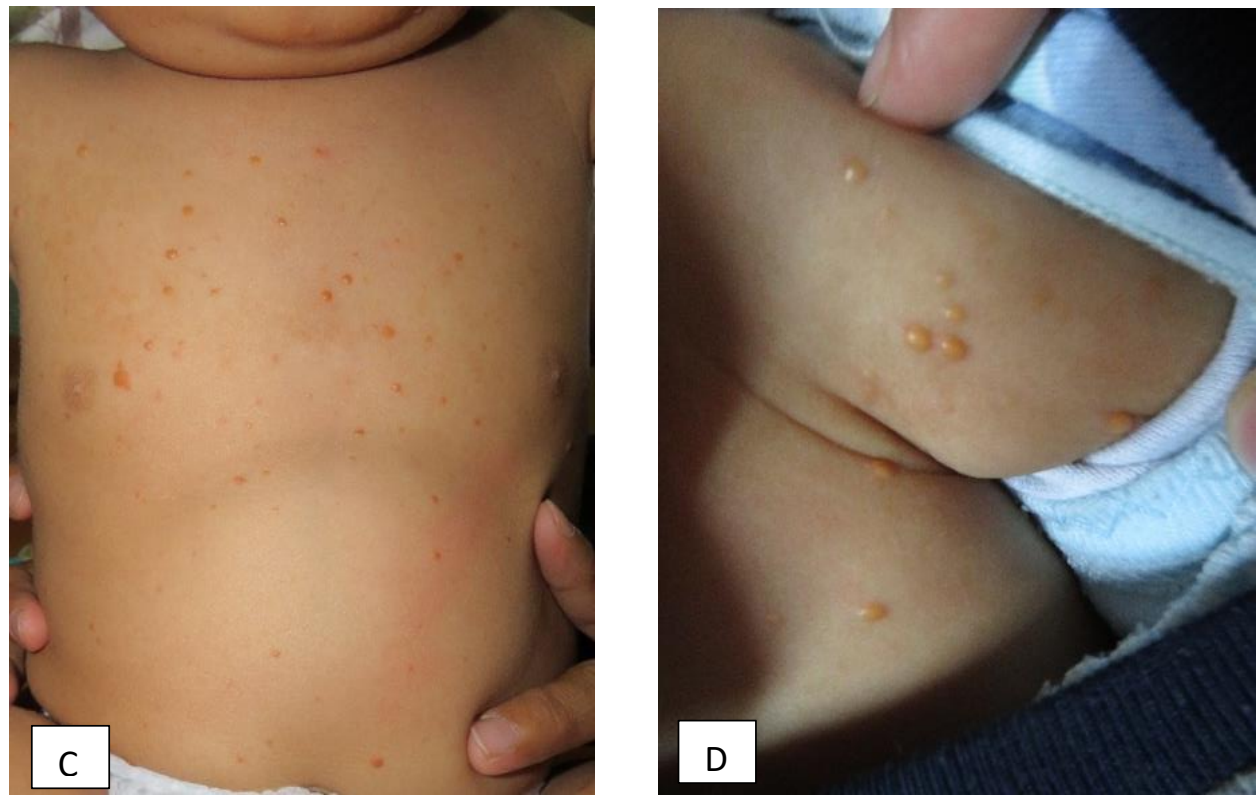

Figure 2. Yellowish papule on patient chest (C) and arm (D) 




Figure 3. Dermoscopy examination showed C) Multiple pale yellowish papule with sun setting appearance and (D) Branch and linear vessels

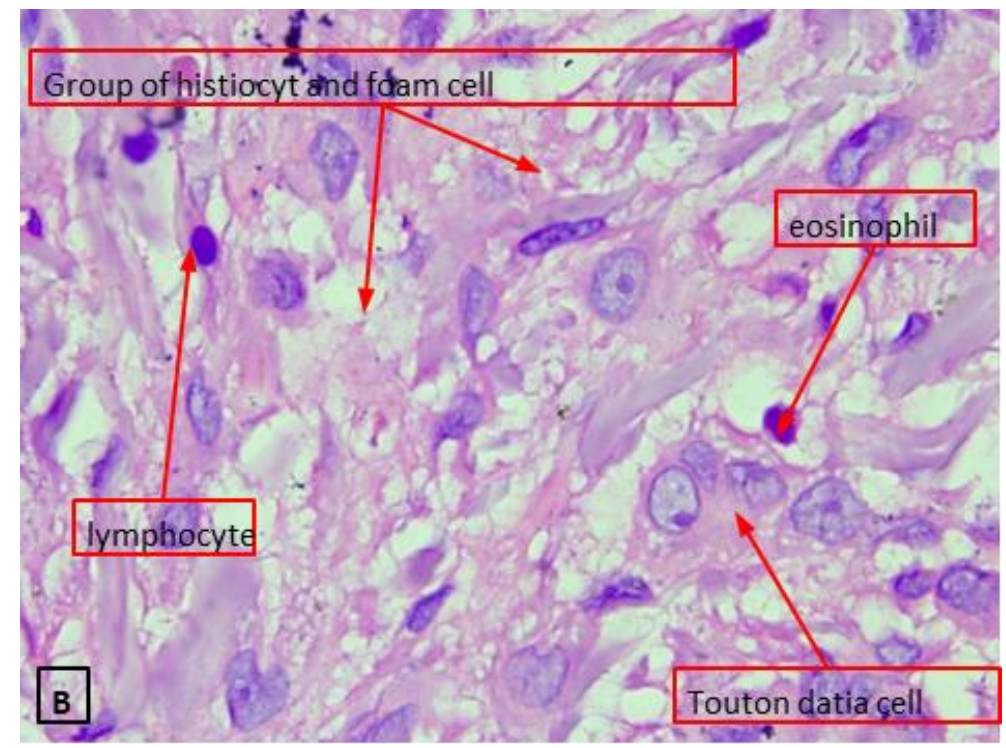

Figure 4. Histhopathology showed accumulation of histiocytes cell in dermis, cell consists of histiocyte cell with spindle type nuclear, a group of foam cell with Touton Giant cell, with infiltrate of eosinophil and lymphocyte.

\section{Discussion}

We report a rare case of 8 months old baby boy with Juvenile Xanthogranuloma Juvenile xanthogranuloma (JXG) is a non-Langerhans cell histiocytosis of childhood. Due to rarity of non Langerhans cell histyosis, the exact prevalence of juvenile xanthogranuloma remain unknown. ${ }^{1}$ We describe a patient with multiple cutaneous JXG who also developed hyperlipidemia. Histiocytosis encompasses a group of rare heterogeneous diseases of unknown cause. They are characterized by the proliferation of histiocytes and are classified by the component cells that accumulate in the affected tissue or organ. JXG is usually benign, self healing, histiocytic disorder of infancy or childhood that is characterized JXG usually manifests with both solitary and multiple papules or nodules that are usually located on the face, neck, and upper trunk, and on other body parts, including lungs, bone, heart, and GI tract. 2,5

JXG may be clinically subdivided into several forms. The micronodular variant is most common; patients are infants with many papules and small nodules. ${ }^{4}$ This patient is a baby boy aged 8 month that has yellowish papule on the face that gradually increased in size and and number and spread to the trunk, upperlimb and lower limb. Dermoscopy is a noninvasive technique that has been used in the diagnosis of JXG. The pattern described as setting sun is characterized by a yellow- 
orange central area, which may show areas of lighter yellow, correlating with the dermal xanthogranulomatous infiltrate, and an erythematous halo, which may occur at any stage of evolution. ${ }^{4,8} \mathrm{We}$ found yellowish papule and sun setting appearance on dermoscopy examination of this patient.

Laboratory findings revealed elevated total cholesterol at $188 \mathrm{mg} / \mathrm{dL}$ and LDL-C at $137 \mathrm{mg} / \mathrm{dL}$, and trigliserida at $136 \mathrm{mg} / \mathrm{dl}$ with normal liver function test results. Other laboratory findings are in normal limit. JXG has not been routinely associated with hyperlipidemia or errors of lipid metabolism. There are only two cases so far that described elevated lipid serum. The precise pathogenesis of JXG remains unknown. In addition, the mechanism behind the excessive lipidization of macrophages in the absence of hyperlipidemia is unclear.

JXG is diagnosed by clinical findings, and histopathology examination. Avoiding performance of histopathological examinations sometimes leads to misdiagnosis of JXG. A histopathological ex $\neg$ amination is necessary to make an accurate diagnosis of JXG. The histopathology of JXG is used to diagnose the characteristic histopathological features of reactive gran $\neg$ uloma containing foamy histiocytes, foreign body giant cells, Touton giant cells with a surrounding wreath of nuclei, histiocytes, lymphocytes, and eosinophils. We observed these findings in our patient.

Differential diagnoses depend on the clinical characteristic, and may include xanthoma, molluscum contagiosum, hemangioma, and neurofibroma. In this case, we differential diagnosed with eruptive xanthoma due to yellowish hue of lesion. Elevated lipid serum on this patient is not enough to be considered eruptive xanthoma, since even for infant, lipid serum in eruptive xanthoma usually reach $2000 \mathrm{mg} / \mathrm{dl} .^{4}$

In most cases, JXG usually resolves spontaneously over 1 to 5 years. Treatments include surgical excision, $\mathrm{CO} 2$ laser treatment, intralesional steroid injection, cryotherapy, and low-dose radiotherapy. In the case of a resistant or recurring lesion, chemotherapy should be considered.7,10 Since the lesion usually regress spontaneously, this patient was planned to be observe regularly for two years and was adviced to control regularly to pediatric department.

\section{Conclusion}

We reported a case of A 8 months-old patient with Juvenile Xanthogranuloma. Diagnosis was made by anamnesis, physical examination and histhopathological examination. The patient was planned to get strict observasion for two years until the lesion regressed spontaneously.

\section{References}

1. Dehner LP. Juvenile xanthogranuloma in the first decades of life. A clinicopathologic study of 174 cases with cutaneous an extracutaneous manifestations. Am J Surg Pathol 2003; 27: 579-93.

2. Janssen D, Harms D. Juvenile xanthogranuloma in childhood and adolescence: a clinicopathological study of 129 patients from the Kiel pediatric tumor registry. Am J Surg Pathol 2005; 29: 21-8.

3. Haroche J, Abla O. Uncommon histiocytic disorders: Rosai- Dorfman, juvenile xanthogranuloma, and Erdheim-Chester disease. Hematology Am Soc Hematol Educ Program 2015; 2015: 571-8.

4. Szczerkowska-Dobosz A, Kozicka D, PurzyckaBohdan D, et al. Juvenile xanthogranuloma: a rare benign histiocytic disorder. Adv Dermatol Allergol 2014; 31: 197-200.

5. Liang S, Liu YH, Fang K. Juvenile xanthogranuloma with ocular involvement. Pediatr Dermatol 2009; 26: 232-4.

6. Palmer A, Bowling J. Dermoscopic appearance of juvenile xanthogranuloma. Dermatology 2007; 215: 256-9.

7. Martínez-Morán C, Echeverria-García B, Tardio JC, Borbujo J. Ultrasound appearance of juvenile xanthogranuloma. Actas Dermosifiliogr 2017; 108: 683-5.

8. Saumyen De. A rare case of familial chylomicronemia in a two and half month old boy. IOSR Journal of Medical Sciences (IOSR- 
JDMS) 2015; 22: 327-35.

9. Sonoda T, Hashimoto H, Enjoji M. Juvenile xanthogranuloma. Clinicopathologic analysis and immunohistochemical study of 57 patients. Cancer 1985; 56: 2280-6.
10. Zelger B, Cerio R, Orchard G, Wilson-Jones E. Juvenile and adult xanthogranuloma. A histological and immunohistochemical comparison. Am J Surg Pathol 1994; 18: 12635. 